\title{
ORIGIN AND MODE OF OCCURRENCE OF TRACE ELEMENTS IN MARINE OIL SHALE FROM THE SHENGLI RIVER AREA, NORTHERN TIBET, CHINA
}

\author{
XIUGEN FU*, JIAN WANG, YUHONG ZENG, \\ JIANG CHENG, FUWEN TAN \\ Chengdu Institute of Geology and Mineral Resources, Chengdu 610081, China
}

\begin{abstract}
With the aim of better understanding of geochemistry of marine oil shale, 25 samples from the Shengli River area were studied. The concentrations of Sr, $U, R b, P b$, and Th were 1.22 to 3.66 times higher compared to the average concentrations in the crust (Clarke values), while the concentrations of other elements were slightly higher/lower compared to the respective Clarke values. Trace elements including $\mathrm{U}, \mathrm{Pb}$ and Th are important from the environmental point of view. The elements in the Shengli River oil shale may be divided into three groups according to their modes of occurrence, i.e. group $A, B$, and $C$. Group $A$ exhibits a relatively high affinity with organic matter. Group $B$ has weakly positive or slightly negative correlation coefficients with ash yield, while Group $C$ shows high positive correlation coefficients with ash yield and possibly has an inorganic affinity. Elements related to terrigenous origin in the oil shale seams in the Shengli River oil shale have originated from two sources: the Nadi Kangri Formation felsic volcanic rocks and the Suowa Formation limestone.
\end{abstract}

\section{Introduction}

Oil shale, as an alternative energy resource, has received much attention in recent years [1-2]. In China, oil shale was mainly deposited in lacustrine environments, such as Tertiary oil shale in the Maoming and Huadian areas, and Triassic oil shale in the Minhe basin [3]. Marine oil shale is mainly found in the Qiangtang basin, northern Tibet, China [4-8], including the Bilong Co oil shale zone and the Shengli River-Changshe Mountain oil shale zone. These zones represent the largest marine oil shale resource in China.

The Shengli River-Changshe Mountain oil shale zone is located in the southern part of the North Qiangtang depression, northern Tibet plateau, China (Fig. 1a), comprising the Shengli River oil shale and the Changshe

\footnotetext{
* Corresponding author: e-mail fuxiugen@126.com
} 
Mountain oil shale (Fig. 1b). The Shengli River-Changshe Mountain oil shale zone is exposed for a distance of more than $50 \mathrm{~km}$ in the east-west direction and $30 \mathrm{~km}$ in the north-south direction (Fig. 1b). The proven reserves of the Shengli River-Changshe Mountain oil shale deposit have been estimated to exceed 1 billion tonnes [7], being potentially the largest marine oil shale resource in China.

Earlier studies of the Shengli River-Changshe Mountain oil shale were focused on the organic geochemistry [6] and sedimentary environment of the oil shale beds [7]. Only few studies have been made to examine the trace element composition of this oil shale. The knowledge of the distribution and concentration of trace elements in oil shale is important not only for geochemistry but also for studies related to mobilization of potentially hazardous elements from environmental point of view.

The object of the present study is to determine the composition of trace elements, study the sources of sediments and modes of occurrence of the trace elements in the Shengli River oil shale.

\section{Geological setting}

On a large scale, the Tibetan Plateau constitutes a tectonic collage of continental blocks (terranes). From north to south, Tibet is comprised of the Kunlun-Qaidam, Songpan-Ganzi flysch complex, Qiangtang, and Lhasa terranes, which are separated by the east-striking Anyimaqen-KunlunMuztagh, Hoh Xil-Jinsha River and Banggong Lake-Nujiang River suture zones, respectively [9] (Fig. 1c).

The Qiangtang block, bounded by Hoh Xil-Jinsha River suture zone to the north and Banggong Lake-Nujiang River suture zone to the south, respectively, consists of the South Qiangtang depression, the central uplift and the North Qiangtang depression (Fig. 1a). At the beginning of the Cretaceous period, the Banggong ocean was closed by the northward subduction beneath the Qiangtang terrane, resulting in a large-scale regression in the Qiangtang basin. During this interval, the South Qiangtang depression was uplifted entirely, while the North Qiangtang depression was still a depositional area.

The Shengli River oil shale is located in the southern part of the North Qiangtang depression, northern Tibet plateau, China (Fig. 1a), where Lower Cretaceous marine deposits are widely spread [7], including the Upper part of the Shengli River Formation, the Xueshan Formation and the Bilong Binghe Formation. On the basis of sedimentary structures, lithology and fossils three facies associations were recognized: fluvial-delta, tidal flatlagoon and shallow marine.

Fluvial-delta facies association is represented by the Xueshan Formation in the Qiangtang basin. The Xueshan Formation is mainly composed of sandstone and mudstone with minor conglomerate. Tidal flat-lagoon facies 
association occurs in the Shengli River Formation, and is characterized by oil shale, marl and gypsum salt intercalated with micritic limestone and coquina. Shallow marine facies association is represented by the Bailong Binghe Formation, mainly comprising marl, mudstone, calcarenite and shale.

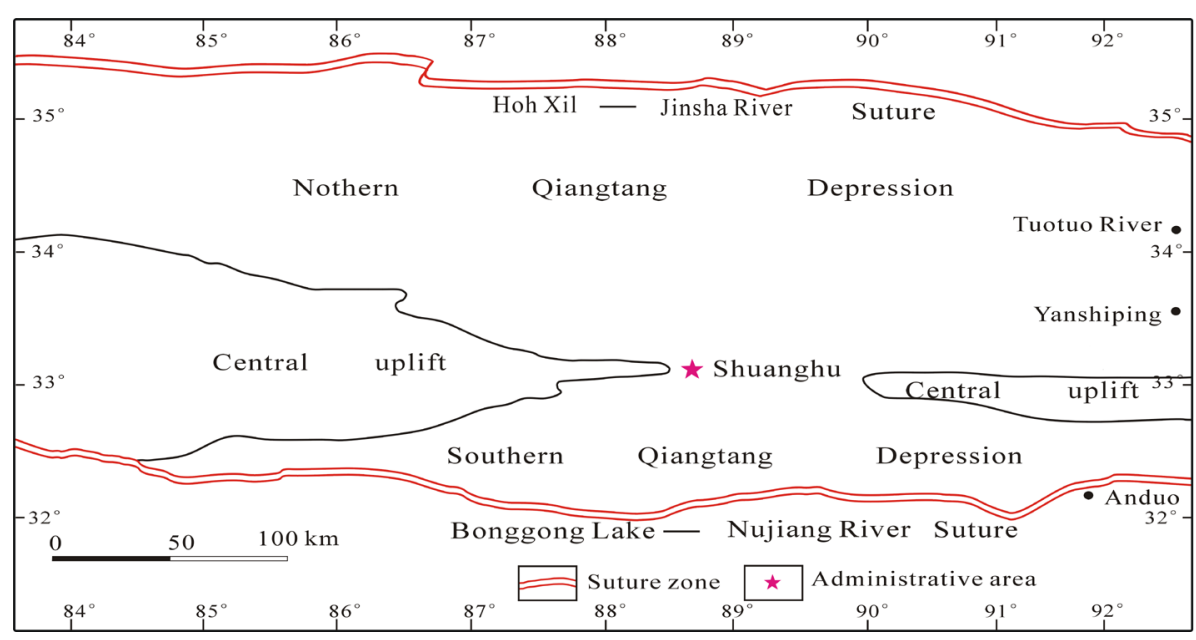

Fig. 1a. Generalized map, showing location of study area.

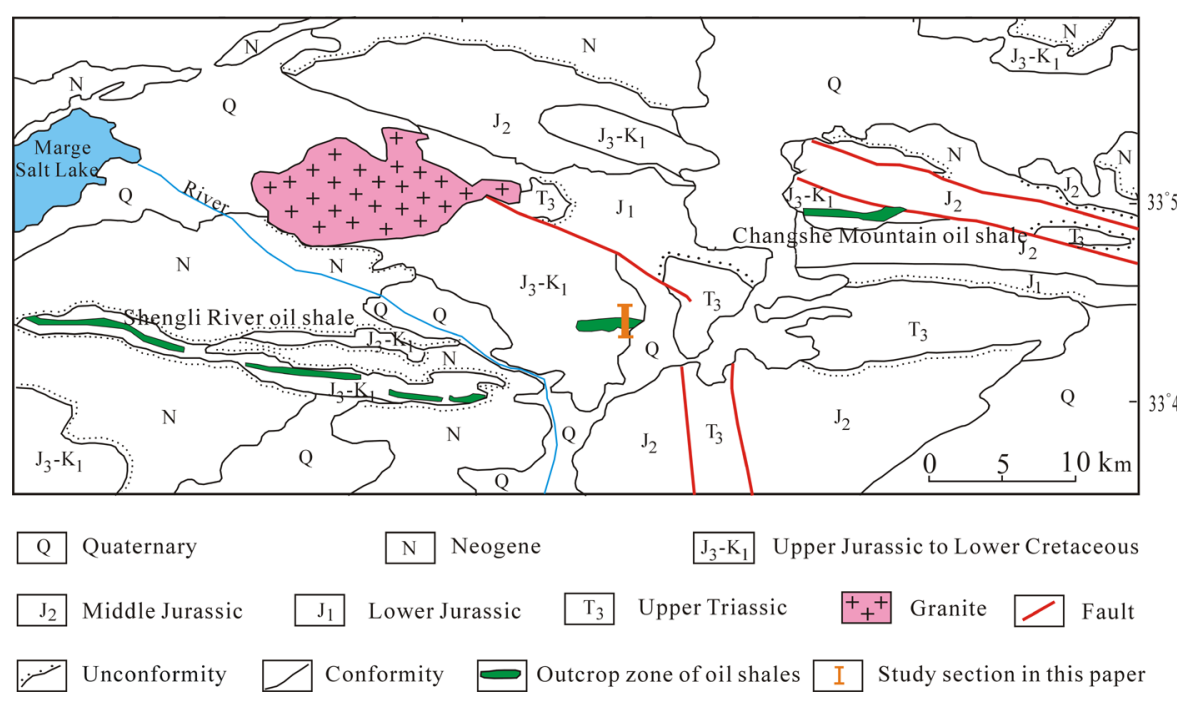

Fig. 1b. Simplified geological map of the Shengli River area, showing location of the oil shale section. 


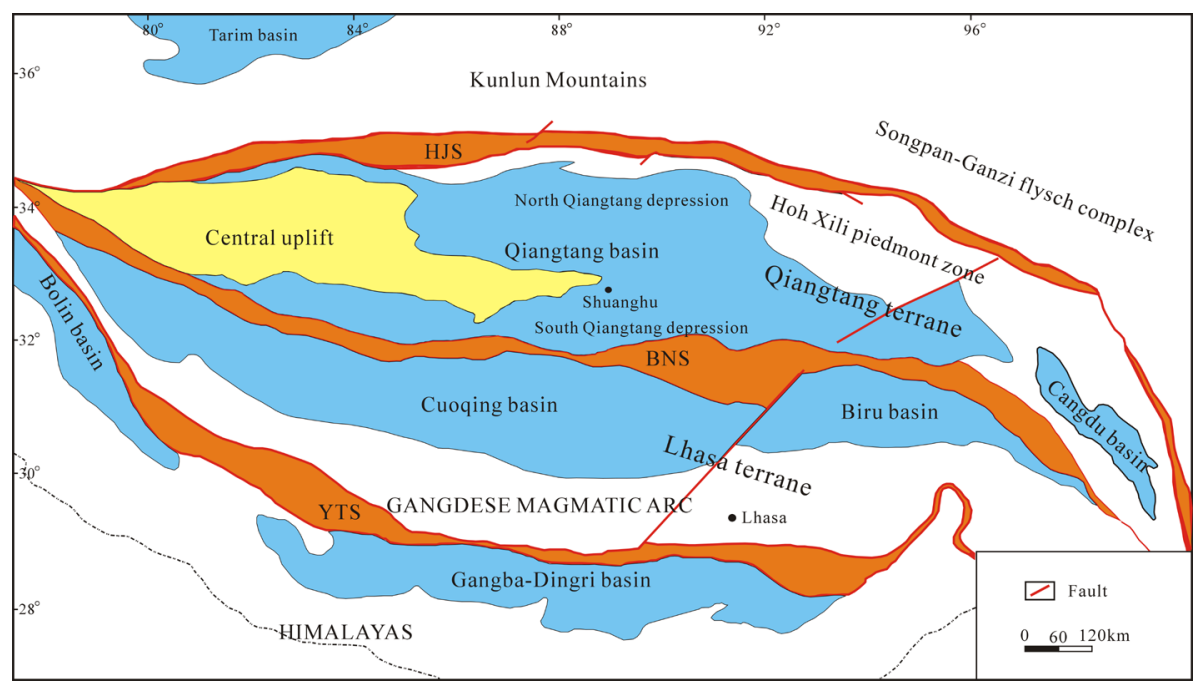

Fig. 1c. Map of the Tibetan plateau showing major Terranes [9].

HJS - Hoh Xil-Jinsha River suture; BNS - Banggong Lake-Nujiang River suture; YTS - Yarlung Tsangpo suture.

\section{Samples and analytical methods}

The study area and sampling section are presented in Fig. 1b. All 25 samples were collected from the Shengli River oil shale section. Twenty of them were collected from oil shale seams with a vertical sampling interval of $0.5 \mathrm{~m}$ on average, and the other five samples were collected from marl layers. Details of sampling location and rock assemblages are shown in Fig. 5.

Samples for geochemical analysis were all crushed and ground to a particle size smaller than 200 mesh. X-ray fluorescence spectrometry (XRF) was used to determine the oxides of major elements, including $\mathrm{Si}, \mathrm{Al}, \mathrm{Ca}, \mathrm{K}, \mathrm{Na}$, $\mathrm{Fe}, \mathrm{Mn}, \mathrm{Mg}$, Ti and $\mathrm{P}$. The analytical procedures are similar to those described by Kimura [10]. The analytical inaccuracy is usually $<5 \%$. Inductively coupled plasma mass spectrometer (ICP-MS) was used to determine the content of trace elements in samples, following the method described in Chinese National Standard DZ/T0223-2001. The samples were digested in $\mathrm{HF}+\mathrm{HNO}_{3}$ using microwave furnace. The level of detection limit for elements is $n \times 10^{-13}-n \times 10^{-12}$, where $n=1-9$ [11].

Ash yield (the ash yield is measured at $815^{\circ} \mathrm{C}$ ) and the content of total sulphur were determined according to Chinese standard methods GB/T2122008 and GB/T214-2007, respectively. Total organic carbon (TOC) and organic sulphur $\left(\mathrm{S}_{\mathrm{o}, \mathrm{d}}\right)$ were determined at the Geological Laboratory of Exploration and Development Research Institute of PetroChina Southwest 
Oil and Gas Field Company, according to Chinese standard methods GB/T19145-2003 and GB/T215-2003, respectively.

The mineral phases were determined by optical microscopic observation and powder X-ray diffraction spectrometer (XRD). The XRD measurements were carried out at Tianjin Institute of Geology and Mineral Resources using a D8 ADVANCE diffractometer equipped with a Cu-target tube and a curved graphite monochromator, operating at $35 \mathrm{kV}$ and $40 \mathrm{~mA}$. Samples were scanned from $5^{\circ}$ to $70^{\circ}$ with a step size of $0.02^{\circ}(2 \theta)$. The analytical procedures follow the method described by Chinese National standard SY/T6210-1996.

The results were statistically analyzed using the Statistical Product and Service Solutions (SPSS) statistical program. Pearson's correlation coefficients between the concentration of the trace elements and ash, main element and sulphur were obtained. The elemental associations were studied by cluster analysis.

\section{Results and discussion}

\section{Characterization of oil shale}

The Shengli River oil shale samples show higher concentration of organic matter (9.42-15.38\%) compared to the marl samples (0.63-2.32\%) (Table 1). The $\mathrm{S}_{\mathrm{o}, \mathrm{d}}$ contents of oil shale samples from the Shengli River area range between $0.61 \%$ and $2.13 \%$, whereas marl samples contain $0.39-0.69 \%$ of organic sulphur (Table 1).

The Shengli River oil shale samples are characterized by high ash yields ranging from $56.40 \%$ to $64.58 \%$ (Table 1) with low or moderate total sulphur $\left(\mathrm{S}_{\mathrm{t}, \mathrm{d}}\right)$ content $(0.75-2.34 \%)$ (Table 1$)$ and intermediate shale oil content (3.60-16.30\%) [8].

\section{Mineralogy of the Shengli River oil shale}

Microscopic observation reveals that in general, the Shengli River oil shale samples contain more than $50 \%$ of minerals by volume $(51.3-72.5 \%)$. These comprise mainly carbonates $(22.5-44.3 \%)$, quartz $(7.0-16.3 \%)$, clay minerals $(16.2-36.5 \%)$ and pyrite $(0.4-3.3 \%)$. The content of calcite is high in the samples of the Shengli River oil shale, where it occurs mainly as fine particles (Fig. 2). Abundant fossil shells were also found in the oil shale samples. The shell material was found to be nearly pure calcite. Clay minerals are common in the oil shale samples. They occur in thin-layered and massive forms (Fig. 2). Quartz occurs mainly as fine particles (Fig. 2).

The minerals identified by X-ray diffraction were calcite $(46.2-77.6 \%)$, clay minerals (mainly illite, also some kaolinite) (14.4-35.4\%), quartz $(5.4-12.6 \%)$ and minor quantities of pyrite (0.7-3.0\%) (Fig. 3). Dolomite $(0-5.6 \%)$ and haematite $(0-2.3 \%)$ were also detected in several oil shale samples. 


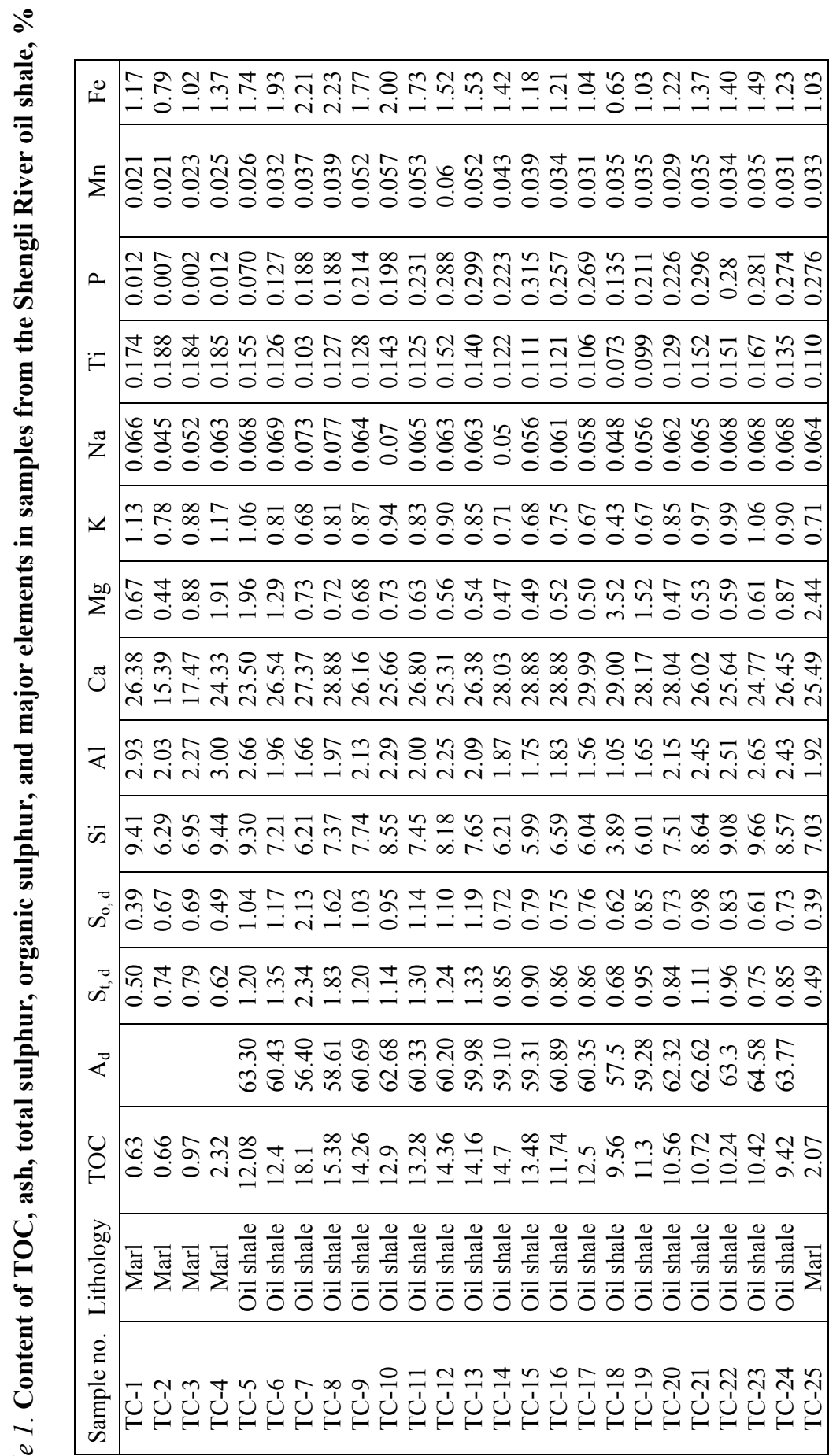




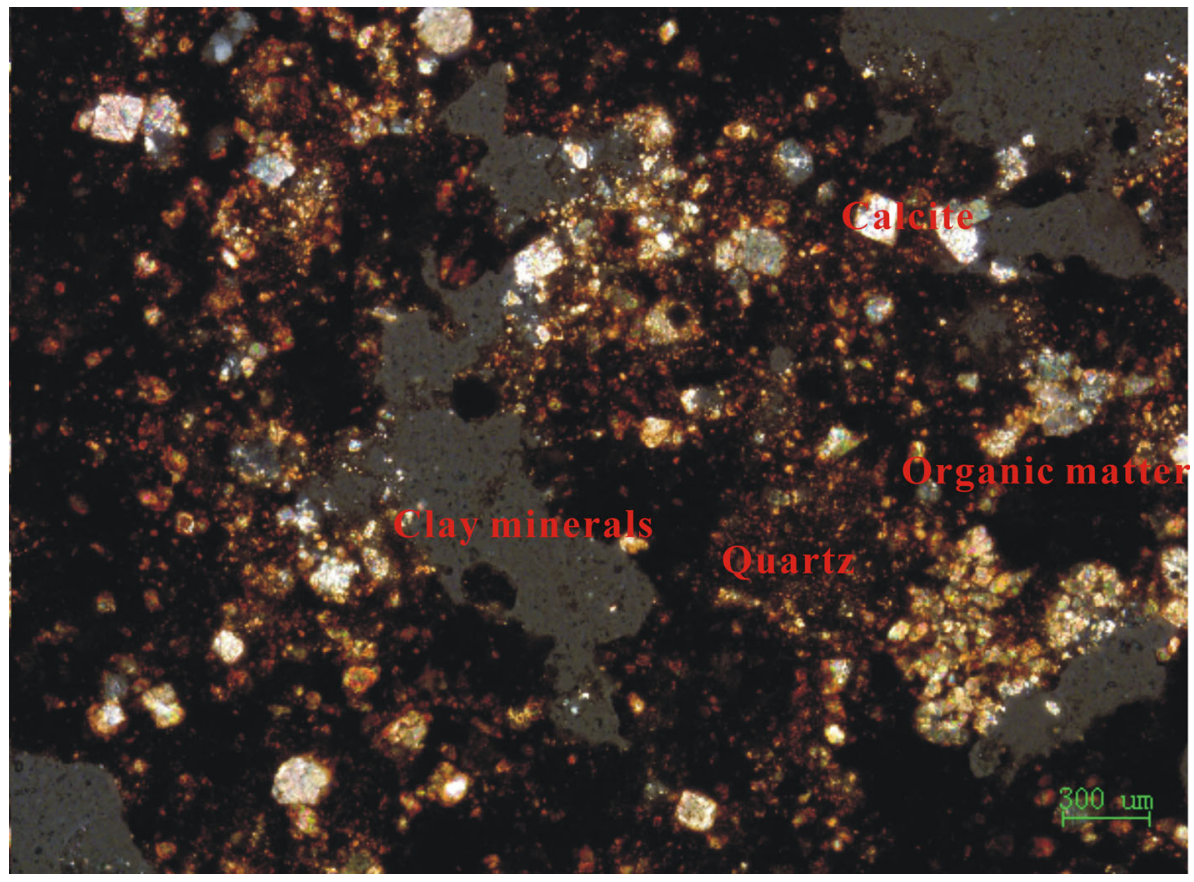

Fig. 2. Selected microscopic photograph showing major petrographical constituents in the Shengli River oil shale samples. Calcite occurring as fine particles, clay minerals occurring in massive form, and quartz occurring mainly as cell-fillings.

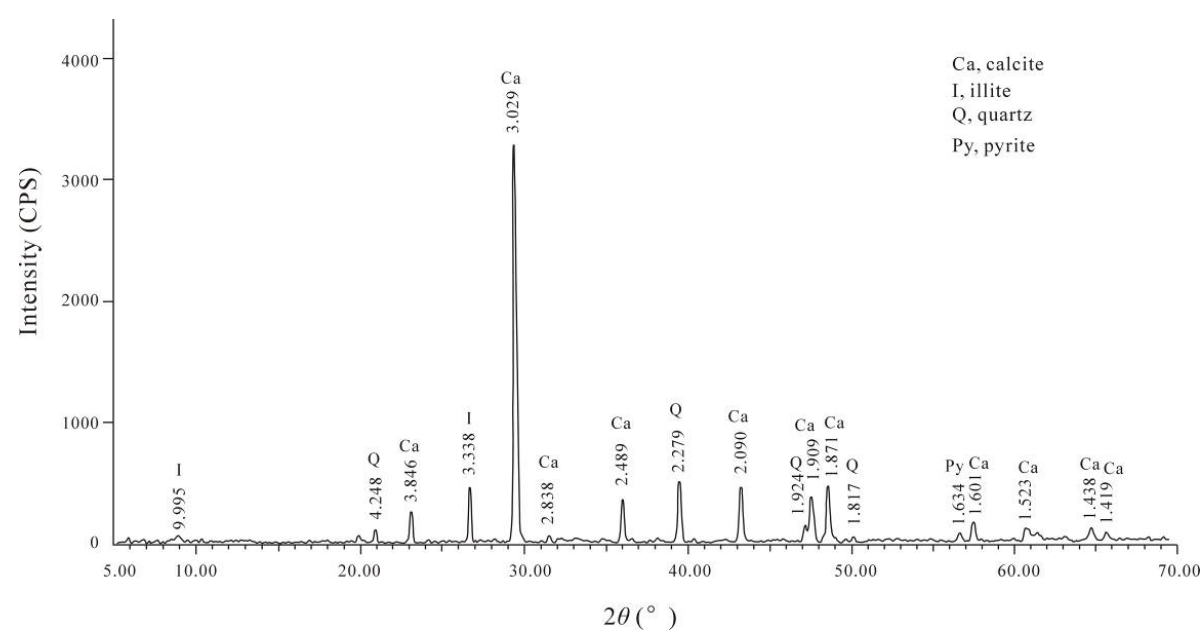

Fig. 3. XRD patterns for selected oil shale sample from the Shengli River oil shale. 


\section{Elements in oil shale}

\section{Geochemistry of main elements}

The most abundant elements in the oil shale samples are $\mathrm{Ca}, \mathrm{Si}$, and $\mathrm{Al}$ (Table 1). High Ca content may refer to abundant calcite, bivalve and gastropod fossil remains in the oil shale seams. Dolomite has also been found in some oil shale samples, indicating that $\mathrm{Ca}$ is partly present in dolomite. High content of $\mathrm{Al}(1.05-2.66 \%)$ and $\mathrm{Si}(3.89-9.66 \%)$ refers that oil shale samples of the Shengli River are rich in clay minerals and quartz. XRD analysis identified great amounts of kaolinite, illite, and quartz. Fe and $\mathrm{K}$ are the second most abundant elements (Table 1), while all other major elements (Mg, $\mathrm{Na}, \mathrm{Ti}, \mathrm{P}$, and $\mathrm{Mn}$ ) have concentration up to $1.0 \%$. In contrast, samples exhibit a slightly higher Si (6.29-9.41\%), Al (1.92-3.00\%), K (0.71-1.17\%) and slightly lower $\mathrm{Ca}(15.39-26.38 \%)$ and $\mathrm{Fe}(0.79-1.37 \%)$ concentrations, while all other elements ( $\mathrm{Mg}, \mathrm{Na}, \mathrm{Ti}, \mathrm{P}$ and $\mathrm{Mn}$ ) have concentration up to $1.0 \%$.

\section{Concentration of trace elements in the Shengli River oil shale}

The concentrations of trace elements of 25 samples from the Shengli River oil shale are presented in Table 2. Table 2 also contains the Clarke values of trace elements and enrichment factor (EF) of some elements. The arithmetic mean for each element from the oil shale samples and the marl samples was calculated.

On the average, the most abundant trace elements are $\mathrm{Sr}$ (407.2$2365.2 \mu \mathrm{g} / \mathrm{g})$ and $\mathrm{Ba}(85.84-256.3 \mu \mathrm{g} / \mathrm{g})$, whereas all the other elements occur in amounts smaller than $100 \mu \mathrm{g} / \mathrm{g}$. Compared to the oil shale samples, the marl samples contain a little lower concentrations of $\mathrm{Co}, \mathrm{Cu}, \mathrm{Ni}, \mathrm{Pb}, \mathrm{Sr}, \mathrm{U}$, and $\mathrm{V}$.

The pioneering work of Patterson et al. [12] established that many trace elements are well above normal abundances in oil shales. Abundance of an element in oil shale may be described by an EF, which is the ratio of the concentration of an element in oil shale to the average concentration in the crust (Clarke value) [13]. The trace elements $\mathrm{Sr}$ and $\mathrm{U}$ can be described by enrichment factor of 3.66 and 2.51, respectively. The abundance of $U$ is bound up with high content of organic matter and abundant pyrite found in oil shale seams because $U$ is typically present in sulphides and/or organic matter [14]. High concentration of Sr may be due to its substitution for $\mathrm{Ca}$ in calcite [15]. The elements $\mathrm{Rb}, \mathrm{Pb}$, and Th have $\mathrm{EF}>1.2$ because $\mathrm{Rb}, \mathrm{Pb}$, and Th are partly controlled by sulphides and/or organic matter. Cobalt, $\mathrm{Cr}, \mathrm{Sc}$, Dy, Er, Eu, Ga, Hf, Ho, Lu, Nb, Sc, Ta, Tb, V, Y, Yb, Zn, and Zr have an EF less than 0.5 . The low concentration of these elements is probably attributed to weathering of source rocks because they are mainly or partly present in detrital clay minerals. All other elements studied show more or less the same concentration as the Clarke values with the EF between 1.2 and 0.5. Clearly, the abundance or concentration of an element may be a function of that association and the origins of the various oil shale fractions. 
Table 2. Concentrations of trace elements in the samples from the Shengli River oil shale, $\mu \mathrm{g} / \mathrm{g}$

\begin{tabular}{|c|c|c|c|c|c|c|c|c|c|}
\hline Element & TC-1 & TC-2 & TC-3 & TC-4 & TC-5 & TC-6 & TC-7 & TC-8 & TC-9 \\
\hline $\mathrm{Ba}$ & 161 & 151.7 & 144.9 & 151.8 & 243.6 & 199 & 130.5 & 150.1 & 229.8 \\
\hline $\mathrm{Ce}$ & 28.7 & 29.49 & 29.5 & 29.09 & 27.62 & 23.38 & 20.62 & 23.68 & 22.99 \\
\hline Co & 6.367 & 8.139 & 6.969 & 6.858 & 11.52 & 12.2 & 12.56 & 12.50 & 9.693 \\
\hline $\mathrm{Cr}$ & 43.93 & 49.08 & 49.84 & 48.31 & 45.36 & 39.44 & 29.35 & 35.09 & 38.9 \\
\hline $\mathrm{Cu}$ & 14.99 & 16.22 & 16.29 & 16.16 & 75.91 & 68.43 & 64.38 & 66.38 & 51.60 \\
\hline Dy & 1.609 & 1.573 & 1.685 & 1.537 & 1.633 & 1.526 & 1.468 & 1.568 & 1.526 \\
\hline Er & 0.953 & 0.942 & 0.921 & 0.887 & 0.889 & 0.86 & 0.764 & 0.833 & 0.865 \\
\hline $\mathrm{Eu}$ & 0.431 & 0.416 & 0.441 & 0.464 & 0.427 & 0.415 & 0.39 & 0.394 & 0.380 \\
\hline $\mathrm{Ga}$ & 9.033 & 10.23 & 9.88 & 9.377 & 8.577 & 6.826 & 5.823 & 6.921 & 6.540 \\
\hline $\mathrm{Gd}$ & 1.943 & 1.867 & 1.905 & 1.85 & 1.893 & 1.718 & 1.72 & 1.792 & 1.662 \\
\hline $\mathrm{Ge}$ & 0.643 & 0.662 & 0.715 & 0.683 & 0.735 & 0.648 & 0.581 & 0.635 & 0.731 \\
\hline Hf & 1.612 & 1.792 & 1.709 & 1.719 & 1.499 & 1.154 & 0.992 & 1.170 & 1.218 \\
\hline Ho & 0.331 & 0.315 & 0.326 & 0.327 & 0.328 & 0.308 & 0.301 & 0.320 & 0.311 \\
\hline $\mathrm{La}$ & 13.63 & 14.23 & 14.37 & 14.38 & 13.07 & 11.25 & 10.17 & 11.55 & 11.54 \\
\hline $\mathrm{Lu}$ & 0.136 & 0.148 & 0.14 & 0.142 & 0.138 & 0.121 & 0.105 & 0.121 & 0.126 \\
\hline $\mathrm{Nb}$ & 5.986 & 6.466 & 6.413 & 6.213 & 5.272 & 4.228 & 3.467 & 4.308 & 4.324 \\
\hline $\mathrm{Nd}$ & 11.72 & 11.77 & 12.08 & 12.06 & 11.26 & 9.755 & 8.882 & 9.853 & 9.562 \\
\hline $\mathrm{Ni}$ & 32.38 & 35.97 & 33.51 & 36.95 & 69.48 & 64.98 & 64.1 & 71.07 & 57.47 \\
\hline $\mathrm{Pb}$ & 7.053 & 7.94 & 8.504 & 7.407 & 16.1 & 16.82 & 13.76 & 16.41 & 13.07 \\
\hline $\operatorname{Pr}$ & 3.356 & 3.369 & 3.409 & 3.443 & 3.191 & 2.743 & 2.46 & 2.75 & 2.671 \\
\hline $\mathrm{Rb}$ & 69.21 & 77.41 & 75.67 & 74.07 & 65.32 & 50.39 & 39.25 & 49.08 & 50.22 \\
\hline $\mathrm{Sc}$ & 7.058 & 7.811 & 7.752 & 7.297 & 6.868 & 5.475 & 4.403 & 5.599 & 5.760 \\
\hline $\mathrm{Sm}$ & 2.221 & 2.194 & 2.182 & 2.247 & 2.126 & 1.96 & 1.771 & 1.953 & 1.837 \\
\hline $\mathrm{Sr}$ & 446.6 & 428.5 & 436 & 407.2 & 482.7 & 557.1 & 561.1 & 605.0 & 2365.2 \\
\hline $\mathrm{Ta}$ & 0.413 & 0.439 & 0.434 & 0.433 & 0.392 & 0.301 & 0.248 & 0.307 & 0.332 \\
\hline $\mathrm{Tb}$ & 0.286 & 0.291 & 0.294 & 0.284 & 0.301 & 0.282 & 0.255 & 0.29 & 0.273 \\
\hline $\mathrm{Th}$ & 5.294 & 5.865 & 5.645 & 5.21 & 5.42 & 4.337 & 3.482 & 4.214 & 4.332 \\
\hline $\mathrm{Tm}$ & 0.132 & 0.149 & 0.136 & 0.136 & 0.128 & 0.120 & 0.110 & 0.120 & 0.134 \\
\hline $\mathrm{U}$ & 1.245 & 1.534 & 1.388 & 1.342 & 3.564 & 3.542 & 3.478 & 3.403 & 2.331 \\
\hline $\mathrm{V}$ & 55.58 & 60.56 & 60.78 & 68.18 & 96.2 & 95.11 & 86.34 & 92.46 & 78.09 \\
\hline Y & 9.57 & 9.546 & 9.628 & 9.323 & 9.738 & 9.146 & 8.56 & 9.398 & 9.451 \\
\hline $\mathrm{Yb}$ & 0.888 & 0.913 & 0.919 & 0.922 & 0.852 & 0.765 & 0.692 & 0.789 & 0.823 \\
\hline $\mathrm{Zn}$ & 22.58 & 21.69 & 23.46 & 62.41 & 36.99 & 27.57 & 20.93 & 24.87 & 25.12 \\
\hline $\mathrm{Zr}$ & 63.11 & 67.91 & 65.95 & 62.81 & 55.57 & 44.65 & 36.99 & 45.01 & 44.56 \\
\hline
\end{tabular}

\begin{tabular}{|c|c|c|c|c|c|c|c|c|c|c|c|}
\hline Element & TC-10 & TC-11 & TC-12 & TC-13 & TC-14 & TC-15 & TC-16 & TC-17 & TC-18 & TC-19 & TC-20 \\
\hline $\mathrm{Ba}$ & 171.0 & 171.5 & 164.1 & 141.0 & 138.3 & 128.3 & 152.8 & 168.2 & 85.84 & 132.5 & 207.3 \\
$\mathrm{Ce}$ & 24.75 & 22.95 & 26.92 & 24.64 & 19.32 & 18.76 & 20.74 & 18.73 & 13.73 & 17.09 & 22.51 \\
$\mathrm{Co}$ & 10.94 & 12.07 & 13.26 & 12.7 & 9.988 & 9.501 & 9.472 & 8.367 & 5.144 & 7.611 & 7.474 \\
$\mathrm{Cr}$ & 38.22 & 35.91 & 47.8 & 36.95 & 29.92 & 29.43 & 36.88 & 31.84 & 16.74 & 31.29 & 37.76 \\
$\mathrm{Cu}$ & 58.33 & 57.61 & 78.44 & 69.19 & 59.61 & 58.46 & 47.09 & 40.96 & 20.4 & 35.34 & 43.23 \\
$\mathrm{Dy}$ & 1.657 & 1.613 & 1.911 & 1.830 & 1.490 & 1.63 & 1.486 & 1.476 & 1.038 & 1.285 & 1.653 \\
$\mathrm{Er}$ & 0.958 & 0.910 & 1.033 & 1.029 & 0.867 & 0.921 & 0.867 & 0.843 & 0.616 & 0.755 & 0.946 \\
$\mathrm{Eu}$ & 0.417 & 0.399 & 0.467 & 0.455 & 0.381 & 0.378 & 0.399 & 0.367 & 0.274 & 0.339 & 0.47 \\
$\mathrm{Ga}$ & 7.024 & 6.642 & 8.168 & 7.170 & 5.715 & 5.430 & 5.703 & 5.070 & 3.397 & 4.828 & 6.125 \\
$\mathrm{Gd}$ & 1.846 & 1.797 & 2.072 & 2.084 & 1.726 & 1.769 & 1.734 & 1.656 & 1.230 & 1.465 & 1.968 \\
$\mathrm{Ge}$ & 0.636 & 0.784 & 0.88 & 0.672 & 0.493 & 0.475 & 0.505 & 0.418 & 0.26 & 0.412 & 0.441 \\
$\mathrm{Hf}$ & 1.318 & 1.199 & 1.452 & 1.345 & 1.073 & 1.059 & 1.130 & 1.071 & 0.719 & 0.968 & 1.235 \\
$\mathrm{Ho}$ & 0.343 & 0.326 & 0.382 & 0.378 & 0.310 & 0.330 & 0.312 & 0.302 & 0.218 & 0.286 & 0.345 \\
$\mathrm{La}$ & 12.50 & 11.51 & 13.10 & 12.25 & 9.921 & 9.725 & 10.54 & 9.667 & 6.963 & 8.725 & 11.27 \\
\hline
\end{tabular}




\begin{tabular}{|c|c|c|c|c|c|c|r|c|r|r|c|}
\hline Element & TC-10 & TC-11 & TC-12 & TC-13 & TC-14 & TC-15 & TC-16 & TC-17 & TC-18 & TC-19 & TC-20 \\
\hline $\mathrm{Lu}$ & 0.141 & 0.129 & 0.146 & 0.141 & 0.129 & 0.122 & 0.132 & 0.116 & 0.09 & 0.117 & 0.136 \\
$\mathrm{Nb}$ & 4.788 & 4.255 & 4.989 & 4.481 & 3.423 & 3.404 & 3.944 & 3.592 & 2.422 & 3.335 & 4.107 \\
$\mathrm{Nd}$ & 10.09 & 9.743 & 11.180 & 10.39 & 8.359 & 8.152 & 8.756 & 8.226 & 5.882 & 7.297 & 9.738 \\
$\mathrm{Ni}$ & 60.42 & 63.08 & 74.03 & 72.92 & 66.64 & 61.06 & 60.78 & 56.60 & 41.87 & 50.95 & 53.94 \\
$\mathrm{~Pb}$ & 14.88 & 14.25 & 14.15 & 12.30 & 11.08 & 10.21 & 10.28 & 9.177 & 5.778 & 8.077 & 12.09 \\
$\mathrm{Pr}$ & 2.806 & 2.659 & 3.077 & 2.850 & 2.360 & 2.187 & 2.431 & 2.201 & 1.632 & 1.997 & 2.578 \\
$\mathrm{Rb}$ & 53.75 & 49.29 & 59.04 & 52.66 & 42.11 & 39.86 & 44.30 & 37.99 & 24.93 & 40.27 & 49.96 \\
$\mathrm{Sc}$ & 6.311 & 5.597 & 6.647 & 5.983 & 6.021 & 5.380 & 5.187 & 4.528 & 2.834 & 4.294 & 5.614 \\
$\mathrm{Sm}$ & 1.966 & 1.944 & 2.226 & 2.084 & 1.715 & 1.679 & 1.741 & 1.696 & 1.204 & 1.477 & 1.963 \\
$\mathrm{Sr}$ & 1327 & 966.2 & 627.1 & 695.7 & 1010.5 & 1029.8 & 1095.2 & 964.2 & 990.5 & 1338.6 & 1229.6 \\
$\mathrm{Ta}$ & 0.367 & 0.315 & 0.366 & 0.347 & 0.265 & 0.268 & 0.300 & 0.279 & 0.185 & 0.264 & 0.318 \\
$\mathrm{~Tb}$ & 0.288 & 0.288 & 0.336 & 0.313 & 0.260 & 0.290 & 0.256 & 0.260 & 0.193 & 0.242 & 0.314 \\
$\mathrm{Th}$ & 4.809 & 4.389 & 5.039 & 4.618 & 3.672 & 3.570 & 3.973 & 3.514 & 2.422 & 3.472 & 4.136 \\
$\mathrm{Tm}$ & 0.135 & 0.130 & 0.146 & 0.144 & 0.129 & 0.133 & 0.123 & 0.119 & 0.093 & 0.113 & 0.130 \\
$\mathrm{U}$ & 2.236 & 2.361 & 2.966 & 2.709 & 1.889 & 1.839 & 1.835 & 1.799 & 1.047 & 1.572 & 1.556 \\
$\mathrm{~V}$ & 73.29 & 70.68 & 80.34 & 72.92 & 61.56 & 60.29 & 61.11 & 56.88 & 49.35 & 56.52 & 55.72 \\
$\mathrm{Y}$ & 10.02 & 9.632 & 11.20 & 10.52 & 8.682 & 9.371 & 9.054 & 8.616 & 6.35 & 8.111 & 9.807 \\
$\mathrm{Yb}$ & 0.887 & 0.853 & 0.979 & 0.909 & 0.803 & 0.829 & 0.811 & 0.771 & 0.582 & 0.748 & 0.874 \\
$\mathrm{Zn}$ & 15.66 & 36.93 & 33.66 & 19.67 & 17.63 & 13.97 & 12.03 & 12.05 & 8.09 & 10.08 & 13.60 \\
$\mathrm{Zr}$ & 49.34 & 44.29 & 52.94 & 47.39 & 37.02 & 36.61 & 40.19 & 37.10 & 25.66 & 34.02 & 45.28 \\
\hline
\end{tabular}

\begin{tabular}{|c|c|c|c|c|c|c|c|c|c|c|}
\hline Element & TC-21 & TC-22 & TC-23 & TC-24 & TC-25 & $\begin{array}{l}\text { Clarke } \\
\text { value }^{\mathrm{a}}\end{array}$ & $\mathrm{EF}$ & $\begin{array}{l}\text { WM in } \\
\text { OS }\end{array}$ & $\begin{array}{c}\text { WM in } \\
\text { Marl }\end{array}$ & $\begin{array}{c}\text { Australian } \\
\text { oil shale }^{b}\end{array}$ \\
\hline $\mathrm{Ba}$ & 308.6 & 256.3 & 247.7 & 139.6 & 208.7 & 250 & 0.71 & 178.3 & 163.62 & 300 \\
\hline $\mathrm{Ce}$ & 25.58 & 25.15 & 27.39 & 23.24 & 19.14 & 33 & 0.68 & 22.49 & 27.18 & 26 \\
\hline Co & 9.188 & 9.033 & 10.05 & 7.657 & 6.406 & 29 & 0.35 & 10.05 & 6.95 & 9 \\
\hline $\mathrm{Cr}$ & 38.42 & 44.65 & 50.11 & 40.49 & 33.33 & 185 & 0.2 & 36.73 & 44.9 & 90 \\
\hline $\mathrm{Cu}$ & 50.93 & 44.8 & 50.0 & 36.41 & 30.95 & 75 & 0.72 & 53.88 & 18.92 & 110 \\
\hline Dy & 1.948 & 1.912 & 2.116 & 1.792 & 1.496 & 3.7 & 0.44 & 1.628 & 1.58 & 3.8 \\
\hline Er & 1.145 & 1.096 & 1.164 & 0.987 & 0.828 & 2.2 & 0.42 & 0.917 & 0.91 & 2.3 \\
\hline $\mathrm{Eu}$ & 0.527 & 0.503 & 0.54 & 0.436 & 0.393 & 1.1 & 0.38 & 0.418 & 0.43 & 1 \\
\hline $\mathrm{Ga}$ & 7.141 & 7.221 & 8.058 & 6.267 & 5.096 & 18 & 0.36 & 6.43 & 8.72 & 6 \\
\hline $\mathrm{Gd}$ & 2.233 & 2.214 & 2.40 & 2.023 & 1.729 & 3.3 & 0.56 & 1.85 & 1.86 & 3 \\
\hline $\mathrm{Ge}$ & 0.57 & 0.531 & 0.594 & 0.529 & 0.368 & & & 0.577 & 0.61 & \\
\hline $\mathrm{Hf}$ & 1.527 & 1.467 & 1.733 & 1.333 & 1.165 & 3 & 0.41 & 1.233 & 1.6 & 1 \\
\hline Ho & 0.397 & 0.378 & 0.442 & 0.349 & 0.304 & 0.78 & 0.42 & 0.333 & 0.32 & 0.6 \\
\hline $\mathrm{La}$ & 12.94 & 12.53 & 13.53 & 11.34 & 9.521 & 16 & 0.7 & 11.2 & 13.22 & 18 \\
\hline $\mathrm{Lu}$ & 0.161 & 0.160 & 0.171 & 0.144 & 0.121 & 0.3 & 0.43 & 0.132 & 0.14 & 0.6 \\
\hline $\mathrm{Nb}$ & 4.928 & 4.911 & 5.485 & 4.428 & 3.569 & 11 & 0.38 & 4.2 & 5.73 & 5 \\
\hline $\mathrm{Nd}$ & 11.12 & 10.70 & 11.80 & 9.919 & 8.513 & 16 & 0.6 & 9.53 & 11.23 & 12 \\
\hline $\mathrm{Ni}$ & 57.51 & 58.06 & 62.68 & 50.72 & 47 & 105 & 0.58 & 60.92 & 37.16 & 160 \\
\hline $\mathrm{Pb}$ & 11.72 & 9.761 & 11.30 & 8.536 & 6.907 & 8 & 1.5 & 11.99 & 7.56 & 6 \\
\hline $\operatorname{Pr}$ & 3.093 & 2.963 & 3.284 & 2.712 & 2.297 & 3.9 & 0.67 & 2.63 & 3.17 & 3 \\
\hline $\mathrm{Rb}$ & 58.81 & 58.83 & 67.2 & 52.08 & 41.27 & 32 & 1.54 & 49.27 & 67.53 & 22 \\
\hline $\mathrm{Sc}$ & 6.668 & 6.574 & 7.412 & 5.66 & 4.632 & 30 & 0.19 & 5.64 & 6.91 & 6 \\
\hline $\mathrm{Sm}$ & 2.221 & 2.162 & 2.372 & 2.014 & 1.712 & 3.5 & 0.55 & 1.91 & 2.11 & 3 \\
\hline $\mathrm{Sr}$ & 879.9 & 805.9 & 772.1 & 748 & 703.4 & 260 & 3.66 & 952.57 & 484.34 & 400 \\
\hline $\mathrm{Ta}$ & 0.374 & 0.371 & 0.427 & 0.351 & 0.309 & 1 & 0.32 & 0.319 & 0.41 & \\
\hline $\mathrm{Tb}$ & 0.365 & 0.338 & 0.373 & 0.329 & 0.271 & 0.63 & 0.46 & 0.292 & 0.29 & 0.8 \\
\hline Th & 4.90 & 4.914 & 5.570 & 4.497 & 3.716 & 3.5 & 1.22 & 4.264 & 5.15 & 2.3 \\
\hline $\mathrm{Tm}$ & 0.160 & 0.156 & 0.173 & 0.140 & 0.121 & 0.32 & 0.41 & 0.132 & 0.13 & 0.3 \\
\hline $\mathrm{U}$ & 2.035 & 1.832 & 2.055 & 1.635 & 1.396 & 0.91 & 2.51 & 2.28 & 1.38 & 30 \\
\hline V & 65.78 & 65.59 & 71.95 & 59.26 & 49.78 & 230 & 0.31 & 70.47 & 58.98 & 2000 \\
\hline
\end{tabular}




\begin{tabular}{|l|c|c|c|c|c|c|c|c|c|c|}
\hline Element & TC-21 & TC-22 & TC-23 & TC-24 & TC-25 & $\begin{array}{c}\text { Clarke } \\
\text { value }^{\mathrm{a}}\end{array}$ & EF & $\begin{array}{c}\text { WM in } \\
\text { OS }\end{array}$ & $\begin{array}{c}\text { WM in } \\
\text { Marl }\end{array}$ & $\begin{array}{c}\text { Australian } \\
\text { oil shale }^{\mathrm{b}}\end{array}$ \\
\hline $\mathrm{Y}$ & 11.35 & 10.88 & 11.52 & 9.854 & 8.465 & 20 & 0.48 & 9.56 & 9.31 & 28 \\
$\mathrm{Yb}$ & 1.035 & 1.033 & 1.138 & 0.933 & 0.79 & 2.2 & 0.39 & 0.86 & 0.89 & 3 \\
$\mathrm{Zn}$ & 28.12 & 25.39 & 26.47 & 23.39 & 20.68 & 80 & 0.27 & 21.61 & 30.16 & 800 \\
$\mathrm{Zr}$ & 52.01 & 51.45 & 56.80 & 45.88 & 39.36 & 100 & 0.44 & 44.14 & 59.83 & 50 \\
\hline
\end{tabular}

WM in OS - weighed mean in oil shale samples; WM in Marl - weighed mean in marl samples

${ }^{a}$ Taylor and McKennan [13]

${ }^{b}$ Patterson et al. [19]

\section{Geochemical associations}

The cluster analysis divides elements into three associations (Fig. 4), referred to as group $\mathrm{A}, \mathrm{B}$, and $\mathrm{C}$.

Group $\mathrm{A}$ includes $\mathrm{S}_{\mathrm{o}, \mathrm{d}}, \mathrm{S}_{\mathrm{t}, \mathrm{d}}, \mathrm{U}, \mathrm{V}, \mathrm{Fe}, \mathrm{Pb}, \mathrm{Na}, \mathrm{Cu}, \mathrm{Ni}, \mathrm{TOC}$, and $\mathrm{Co}$ (Fig. 4). The correlation coefficients of $\mathrm{S}_{\mathrm{o}, \mathrm{d}}-\mathrm{S}_{\mathrm{t}, \mathrm{d}}(0.998), \mathrm{U}-\mathrm{V}(0.97)$, and $\mathrm{Cu}-$ $\mathrm{Ni}(0.95)$ are all higher than 0.95 . Elements from this group exhibit a relatively high affinity with organic matter (Table 3 ).

Group B: $\mathrm{Mn}, \mathrm{Sr}, \mathrm{Ca}$, and $\mathrm{Mg}$ are clustered in the second association (Fig. 4). The correlation coefficients of pairs of elements in this association are lower than 0.29 . Elements from this group have negative or weakly positive correlation coefficients with ash yield, ranging from -0.62 to 0.007 (Table 3).

Group C includes Dy, Ho, Tb, Y, Tm, Yb, P, Eu, Gd, Er, Lu, Nd, Pr, Ga, $\mathrm{Sm}, \mathrm{Ce}, \mathrm{La}, \mathrm{Ge}, \mathrm{Zn}, \mathrm{Ba}, \mathrm{Si}, \mathrm{K}, \mathrm{Cr}, \mathrm{Nb}, \mathrm{Zr}, \mathrm{Rb}, \mathrm{Th}, \mathrm{Hf}, \mathrm{Ta}, \mathrm{Sc}$, and Ti. The correlation coefficients of Dy-Ho (0.99), Tb-Y (0.96), Tm-Yb (0.98), Eu-Gd (0.98), Er-Lu (0.96), Nd-Pr (0.99), Ga-Sm (0.94), Ce-La (0.99), Si-K (0.99), Al-Cr (0.91), Nb-Zr (0.995), Rb-Th (0.99), Hf-Ta (0.98), Sc-Ti (0.98) are all higher than 0.90 . Elements from this group have relatively high correlation coefficients with ash yield, ranging from 0.60 to 0.85 (Table 3 ).

\section{Affinity of the elements}

The correlation of the element concentrations with ash yield may provide preliminary information for their organic or inorganic affinity [16]. Elements divided into five groups are classified according to their correlation coefficients with ash yield (Table 3 ).

The first group includes elements $\mathrm{Si}, \mathrm{Al}, \mathrm{K}, \mathrm{Ba}, \mathrm{Ce}, \mathrm{Cr}, \mathrm{Dy}, \mathrm{Er}, \mathrm{Eu}, \mathrm{Gd}$, Hf, Ho, La, Lu, Nb, Nd, Pr, Rb, Sc, Sm, Ta, Tb, Th, Ti, Tm, Y, Yb, and Zr (Table 3 ). Those elements have a very high positive correlation coefficient $(>0.65)$ with ash yield indicating mainly inorganic affinity. All those elements are normally related to aluminosilicate minerals demonstrating that they mainly originate from clay minerals, which are also supported by the occurrence of kaolinite and illite identified by the XRD analysis. Note that the $\mathrm{Al} / \mathrm{Si}$ ratios of oil shale samples are low $(0.26-0.30)$, allowing to suggest that $\mathrm{Si}$ has another source besides clay minerals. The abundant quartz identified by the XRD analysis allows to assume that the extra $\mathrm{Si}$ is present in the form of quartz. 


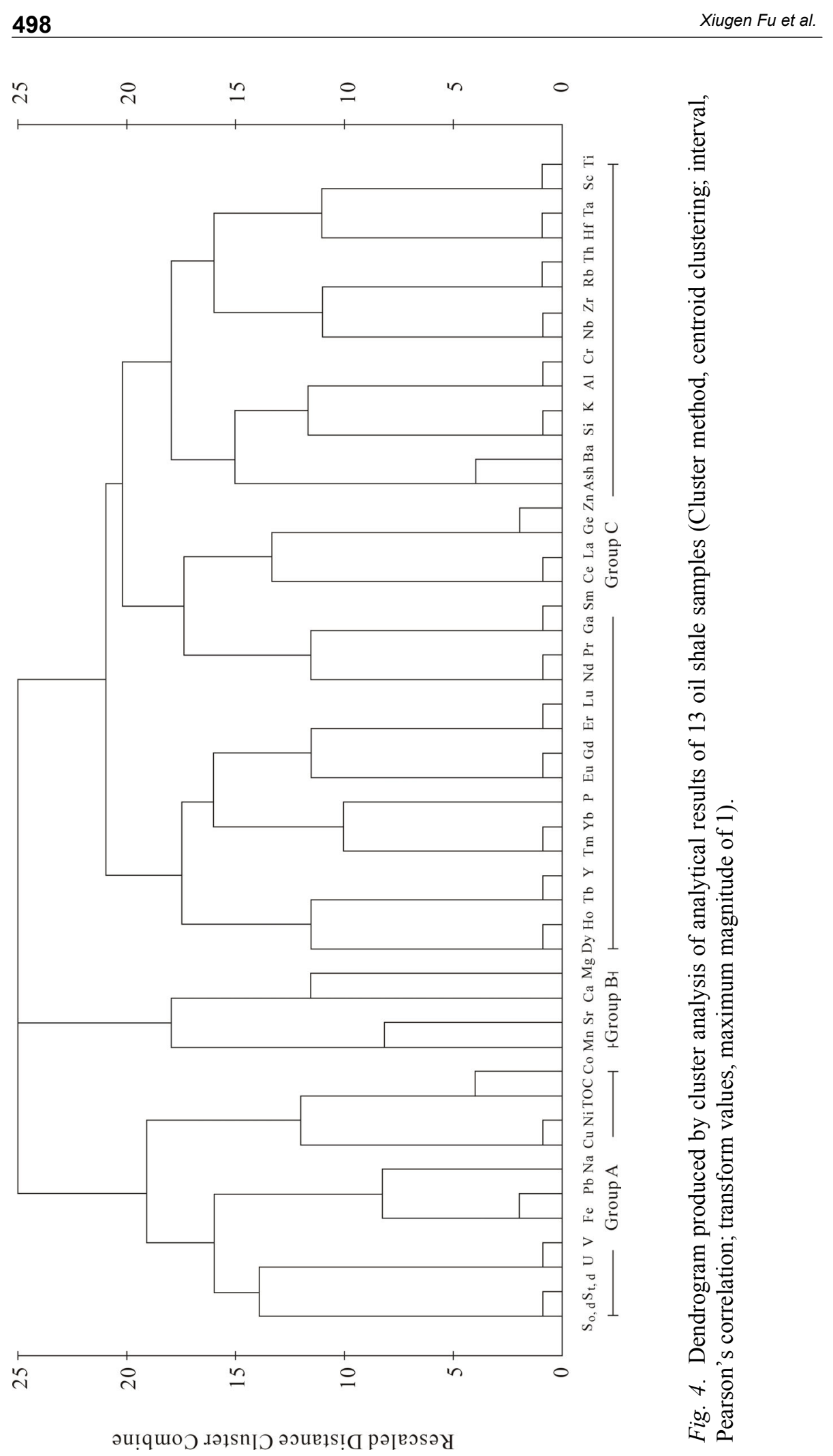


Table 3. Element affinities deduced from the calculation of Pearson's correlation coefficients between the content of trace elements in oil shale and ash yield or selected major elements

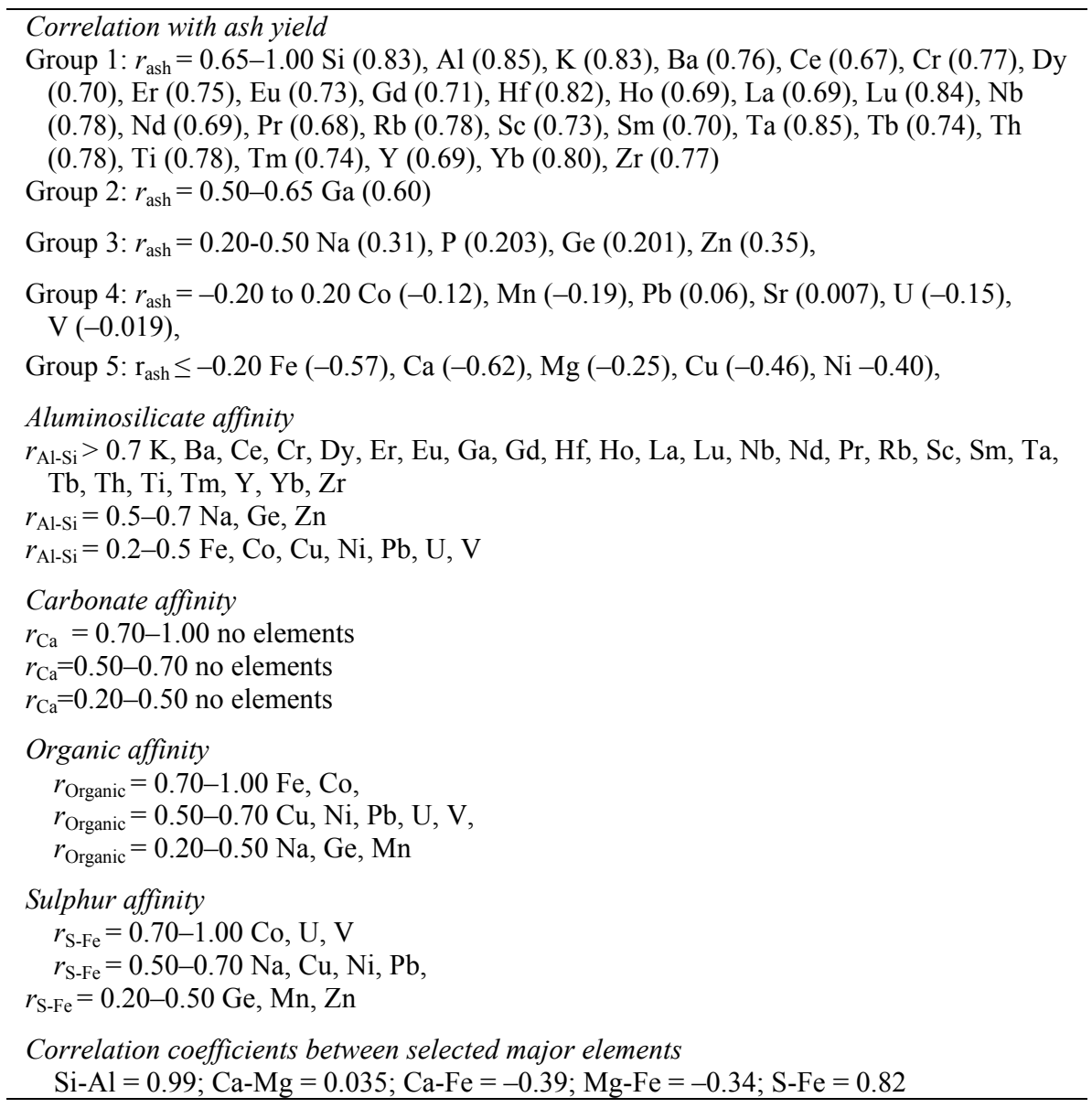

The second group includes element Ga. Gallium displays a positive correlation with ash yield $(r=0.60)$ indicating prevailing inorganic affinity. The significantly positive correlation coefficients between $\mathrm{Ga}$ and $\mathrm{Al}(r=$ $0.89)$; $\mathrm{Si}(r=0.91), \mathrm{K}(r=0.92)$, and $\mathrm{Ti}(r=0.94)$ indicate that Ga is mainly present in clay minerals. Additionally, Ga displays also a weakly positive correlation with $\mathrm{S}_{\mathrm{t}, \mathrm{d}}(r=0.24)$ and appears to be related to the S-bearing minerals such as pyrite.

The correlation coefficients of the elements with ash yield in the third group vary from 0.20 to 0.35 , including elements $\mathrm{Na}, \mathrm{P}, \mathrm{Ge}$, and $\mathrm{Zn}$. Germanium and $\mathrm{Zn}$ exhibit a similar mode of occurrence in the Shengli River oil shale. Both Ge and $\mathrm{Zn}$ are mainly associated with clay minerals. The positive correlations between $\mathrm{Ge}$ and $\mathrm{Al}(r=0.57)$; $\mathrm{Si}(r=0.62), \mathrm{K}(r=$ $0.63)$, and $\mathrm{Ti}(r=0.64) ; \mathrm{Zn}$ and $\mathrm{Al}(r=0.66) ; \mathrm{Si}(r=0.68), \mathrm{K}(r=0.69)$, and Ti $(r=0.67)$ support the above mentioned observations. Additionally, a 
weakly positive correlation between total sulphur, Ge $(r=0.47)$ and $\mathrm{Zn}(r=$ $0.35)$ was also observed in the oil shale seams, indicating a sulphur affinity with Ge and $\mathrm{Zn}$.

The fourth group includes elements with correlation coefficients below the statistically significant value $( \pm 0.2)$ including $\mathrm{Co}, \mathrm{Mn}, \mathrm{Pb}, \mathrm{Sr}, \mathrm{U}$, and $\mathrm{V}$. Cobalt, $\mathrm{Pb}, \mathrm{U}$, and $\mathrm{V}$ exhibit a similar mode of occurrence in the Shengli River oil shale. They are mainly associated with sulphide (probably pyrite) and organic matter. The positive correlations between Co and $\mathrm{S}_{\mathrm{t}, \mathrm{d}}(r=0.71)$; $\mathrm{S}_{\mathrm{o}, \mathrm{d}}(r=0.69)$ and $\mathrm{Fe}(r=0.82) ; \mathrm{Pb}$ and $\mathrm{S}_{\mathrm{t}, \mathrm{d}}(r=0.64) ; \mathrm{S}_{\mathrm{o}, \mathrm{d}}(r=0.61)$ and $\mathrm{Fe}$ $(r=0.89) ; \mathrm{U}^{2}$ and $\mathrm{S}_{\mathrm{t}, \mathrm{d}}(r=0.80) ; \mathrm{S}_{\mathrm{o}, \mathrm{d}}(r=0.78)$ and $\mathrm{Fe}(r=0.85) ; \mathrm{V}$ and $\mathrm{S}_{\mathrm{t}, \mathrm{d}}$ $(r=0.69) ; \mathrm{S}_{\mathrm{o}, \mathrm{d}}(r=0.72)$ and $\mathrm{Fe}(r=0.87)$ support the abovementioned observations. Additionally, a weakly positive correlation between $\mathrm{Al}$ and $\mathrm{Co}$ $(r=0.32) ; \mathrm{Pb}(r=0.42), \mathrm{U}(r=0.27)$ and $\mathrm{V}(r=0.38)$ indicate that $\mathrm{Co}, \mathrm{Pb}$, $\mathrm{U}$, and $\mathrm{V}$ are also partly present in clay minerals. In the Shengli River oil shale, positive relationships have been recorded between $\mathrm{Mn}$ and $\mathrm{S}_{\mathrm{t}, \mathrm{d}}(r=$ $0.22)$, TOC $(r=0.48)$ and $\mathrm{Fe}(r=0.33)$ indicating that $\mathrm{Mn}$ is present in organic matter and S-bearing minerals (probably pyrite).

The elements of the fifth group (Table 3) have negative correlation coefficients with ash yield, which vary between -0.25 and -0.62 ( $\mathrm{Fe}, \mathrm{Ca}, \mathrm{Mg}$, $\mathrm{Cu}$, and $\mathrm{Ni}$ ). The $\mathrm{Ca}$ concentration correlates negatively with ash yield ( $r=$ -0.62 ), allowing to suggest that $\mathrm{Ca}$ is probably present in more than one form, as inferred by Mukhopadhyay et al. [17]. The Shengli River oil shale samples have a high $\mathrm{Ca}$ concentration, which is also supported by the presence of Ca-bearing minerals. Significant amounts of calcite found in oil shale samples support the abovementioned observations. Additionally, oil shale samples TC-8, TC-14, TC-15, TC-16, TC-17, TC-18, TC-19, and TC-20 have high content of $\mathrm{Ca}(28.88 \%, 28.03 \%, 28.88 \%, 28.88 \%, 29.99,28.17$, and $28.04 \%$, respectively) corresponding to abundant gastropod fossil remains, which suggest that $\mathrm{Ca}$ is also related to the fossil remains. $\mathrm{Ni}, \mathrm{Cu}$, and $\mathrm{Fe}$ have a similar mode of occurrence in the Shengli River oil shale. Positive relationships between Fe and $\mathrm{S}_{\mathrm{t}, \mathrm{d}}(r=0.82)$; TOC $(r=0.67), \mathrm{Cu}$ and $\mathrm{S}_{\mathrm{t}, \mathrm{d}}(0.59)$; TOC $(r=$ $0.66)$ and $\mathrm{Fe}(r=0.73)$; Ni and $\mathrm{S}_{\mathrm{t}, \mathrm{d}}(r=0.52)$; TOC $(r=0.50)$ and $\mathrm{Fe}(r=$ 0.66 ) indicate that $\mathrm{Fe}, \mathrm{Cu}$ and $\mathrm{Ni}$ are mainly present in organic matter and S-bearing minerals (probably pyrite). Additionally, a weakly positive correlation between $\mathrm{Al}$ and $\mathrm{Fe}(r=0.35) ; \mathrm{Cu}(r=0.39)$, and $\mathrm{Ni}(r=0.38)$ was also observed in the oil shale seams, indicating an aluminosilicate affinity with $\mathrm{Fe}$, $\mathrm{Cu}$, and $\mathrm{Ni}$.

\section{Environmentally important elements}

In the discussion above, we proposed that abundant elements in the Shengli River oil shale include $\mathrm{Sr}, \mathrm{U}, \mathrm{Rb}, \mathrm{Pb}$, and $\mathrm{Th}$. The elements $\mathrm{U}, \mathrm{Pb}$, and $\mathrm{Th}$ are defined as environmentally important elements [18]. According to Table 3, U and $\mathrm{Pb}$ exhibit mainly organic and sulphur affinity, while Th is mainly associated with the clay minerals in oil shale. Therefore, concentration of Th may play role when oil shale ash is considered to be used as building material. 
The content of $U$ in the Shengli River oil shale samples ranges from 1.047 $\mu \mathrm{g} / \mathrm{g}$ to $3.564 \mu \mathrm{g} / \mathrm{g}$, with an average value of $2.284 \mu \mathrm{g} / \mathrm{g}$, which is approximately 2.5 times higher compared to the Clarke value [13], but lower than $30 \mu \mathrm{g} / \mathrm{g}$ for common Australian oil shale [19] (Table 3). The distributions of $U$ in the Shengli River section are illustrated on Fig. 5. Generally, the upper seams of the Shengli River oil shale are rich in U (e.g., TC-5, TC-6, TC-7, and TC-8), while the middle and lower seams (e.g., TC-17, TC-18, TC-19, and TC-20) show relatively low concentration of $U$. The vertical variations of $U$ show close correlations with TOC and $\mathrm{S}_{\mathrm{t}, \mathrm{d}}$ contents (Fig. 5) suggesting that $\mathrm{U}$ is mainly present in organic matter and S-bearing minerals.

The concentration of $\mathrm{Pb}$ in the oil shale samples ranges from $5.778 \mu \mathrm{g} / \mathrm{g}$ to $16.82 \mu \mathrm{g} / \mathrm{g}$, with an average value of $11.99 \mu \mathrm{g} / \mathrm{g}$, which is approximately 1.5 times higher compared to the Clarke value [13], higher than $6 \mu \mathrm{g} / \mathrm{g}$ for common Australian oil shale [19] (Table 3). The vertical variations of $\mathrm{Pb}$ are similar to that of $\mathrm{U}$. The upper seams have high concentration of $\mathrm{Pb}$ (e.g., TC-5 and TC-6), while the middle and lower seams have relatively low $\mathrm{Pb}$ concentrations (e.g., TC-17, TC-18, and TC-19). The examination of con-

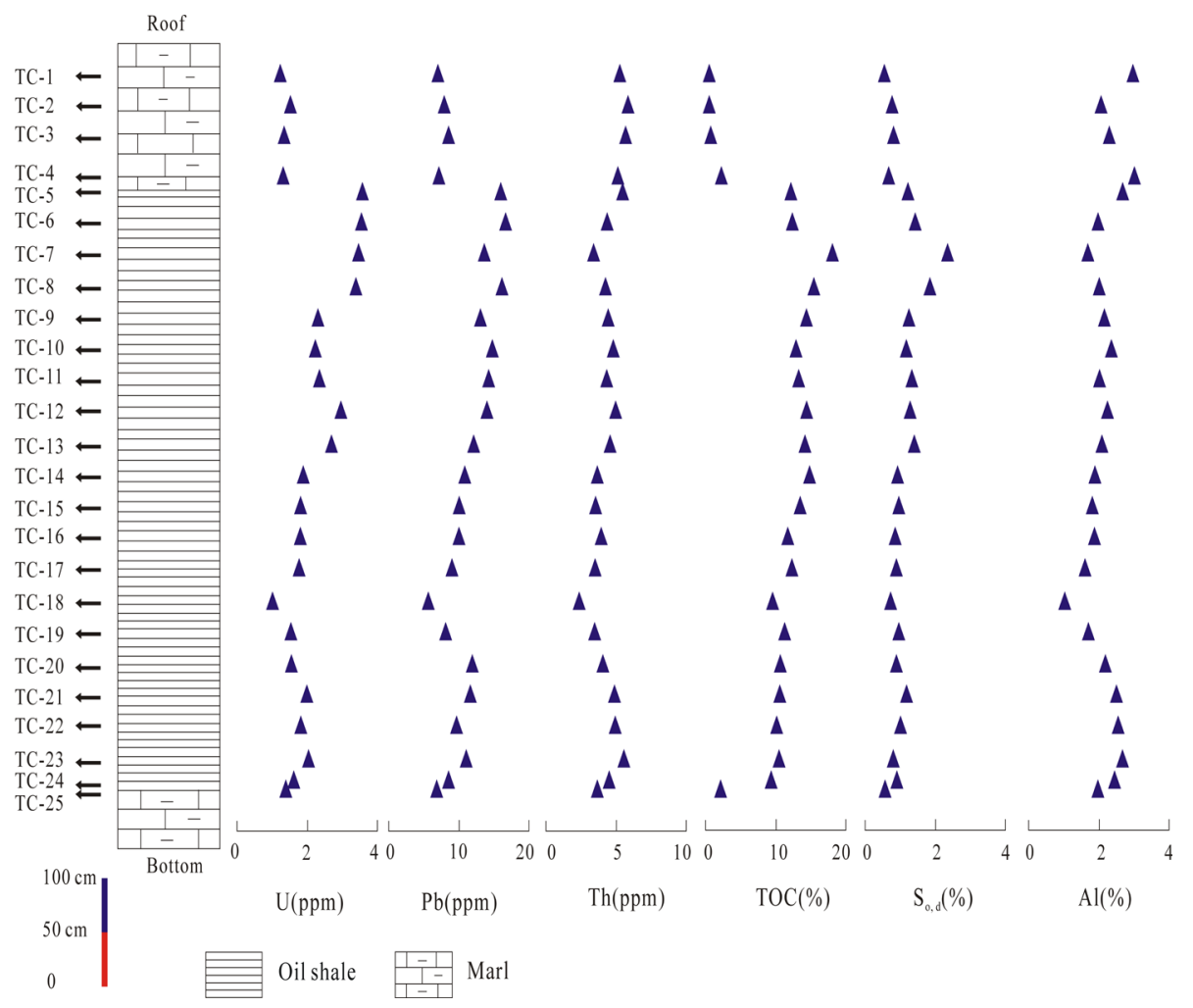

Fig. 5. Vertical variations of total organic carbon (TOC), total sulphur $\left(\mathrm{S}_{\mathrm{o}, \mathrm{d}}\right), \mathrm{Al}$, and environmentally important elements $(\mathrm{U}, \mathrm{Pb}$, and $\mathrm{Th})$ in the Shengli River oil shale section. 
centrations of $\mathrm{Pb}, \mathrm{TOC}$, and $\mathrm{S}_{\mathrm{t}, \mathrm{d}}$ (Fig. 5) in the Shengli River oil shale shows that $\mathrm{Pb}$ is mainly present in organic matter and $\mathrm{S}$-bearing minerals.

The content of Th varies from $2.422 \mu \mathrm{g} / \mathrm{g}$ to $5.570 \mu \mathrm{g} / \mathrm{g}$ in the Shengli River oil shale samples. The weighed mean value is $4.264 \mu \mathrm{g} / \mathrm{g}$, which is approximately 1.2 times higher compared to the Clarke value [13] and higher than $2.3 \mu \mathrm{g} / \mathrm{g}$ for common Australian oil shale [19] (Table 3). High concentration of Th is not uniformly distributed among the seams studied. Very often, high Th concentrations are observed on the lower part (e.g., TC23) and on the top of the seam (e.g., TC-1, TC-2 and TC-3). This trend is in close correlation with variations of $\mathrm{Al}$ concentration (Fig. 6), indicating that clay minerals are probably a Th carriers in the Shengli River oil shale.

\section{Origin of elements in oil shale}

The elements that are positively correlated to the ash in oil shale were probably of terrigenous origin, but those negatively correlated to ash were authigenic [20]. In the Shengli River oil shale, elements $\mathrm{Si}, \mathrm{Al}, \mathrm{K}, \mathrm{Ba}, \mathrm{Ce}$, $\mathrm{Cr}$, Dy, Er, Eu, Gd, Hf, Ho, La, Lu, Nb, Nd, Pr, Rb, Sc, Sm, Ta, Tb, Th, Ti, $\mathrm{Tm}, \mathrm{Y}, \mathrm{Yb}$, and $\mathrm{Zr}$ exhibit high positive correlation coefficients with ash yield indicating mainly terrigenous origin. Elements $\mathrm{Fe}, \mathrm{Ca}, \mathrm{Mg}, \mathrm{Cu}$, and $\mathrm{Ni}$ have negative correlation coefficients with ash yield indicating mainly seawater influence. Other elements, such as $\mathrm{Ge}, \mathrm{Zn}, \mathrm{Co}, \mathrm{Mn}, \mathrm{Pb}, \mathrm{Sr}, \mathrm{U}$, and $\mathrm{V}$, show low or negligible relationship with ash yield indicating they were probably related to seawater and terrigenous source material.

Rare earth elements (REEs) have been used as tracers to identify sources of oil shale mineral matter [8]. The chondrite-normalized REE pattern of the oil shale samples from the Shengli River area is characterized by high LREE/HREE (light rare earth elements/ heavy rare earth elements) ratios (average 7.85), nearly flat HREEs (Fig. 6), and a significantly negative Eu anomaly $(\delta \mathrm{Eu}=0.63-0.72)$. These features characterize a felsic origin [21-22]. Studies by Wang et al. [21] and Fu et al. [22] show that the Nadi Kangri felsic volcanic rocks, widely occurred in the western and eastern parts of the Qiangtang basin, have a relatively high contentof REEs (80.02$220.84 \mu \mathrm{g} / \mathrm{g}$ ) and LREE/HREE ratio (5.94-13.85) and also a distinct or slightly distinctive Eu anomaly (0.42-0.98). The REE distribution patterns in the Nadi Kangri felsic volcanic rocks are similar to those in oil shale samples (Fig. 6), indicating that REEs in the oil shale samples were probably derived from the Nadi Kangri felsic volcanic rocks in the Qiangtang basin.

The Shengli River oil shale was formed at the beginning of the Cretaceous period [5]. During this interval, large-scale regressions could have taken place in the Qiangtang basin region [7]. The depocenter was located in the northwestern part of the North Qiangtang depression. Therefore, the detrital material of oil shale seams in the Shengli River oil shale was mainly derived from the eastern and the southern parts of the Qiangtang basin, where the Upper Jurassic marine deposits (Suowa Formation limestone) are widely spread. 


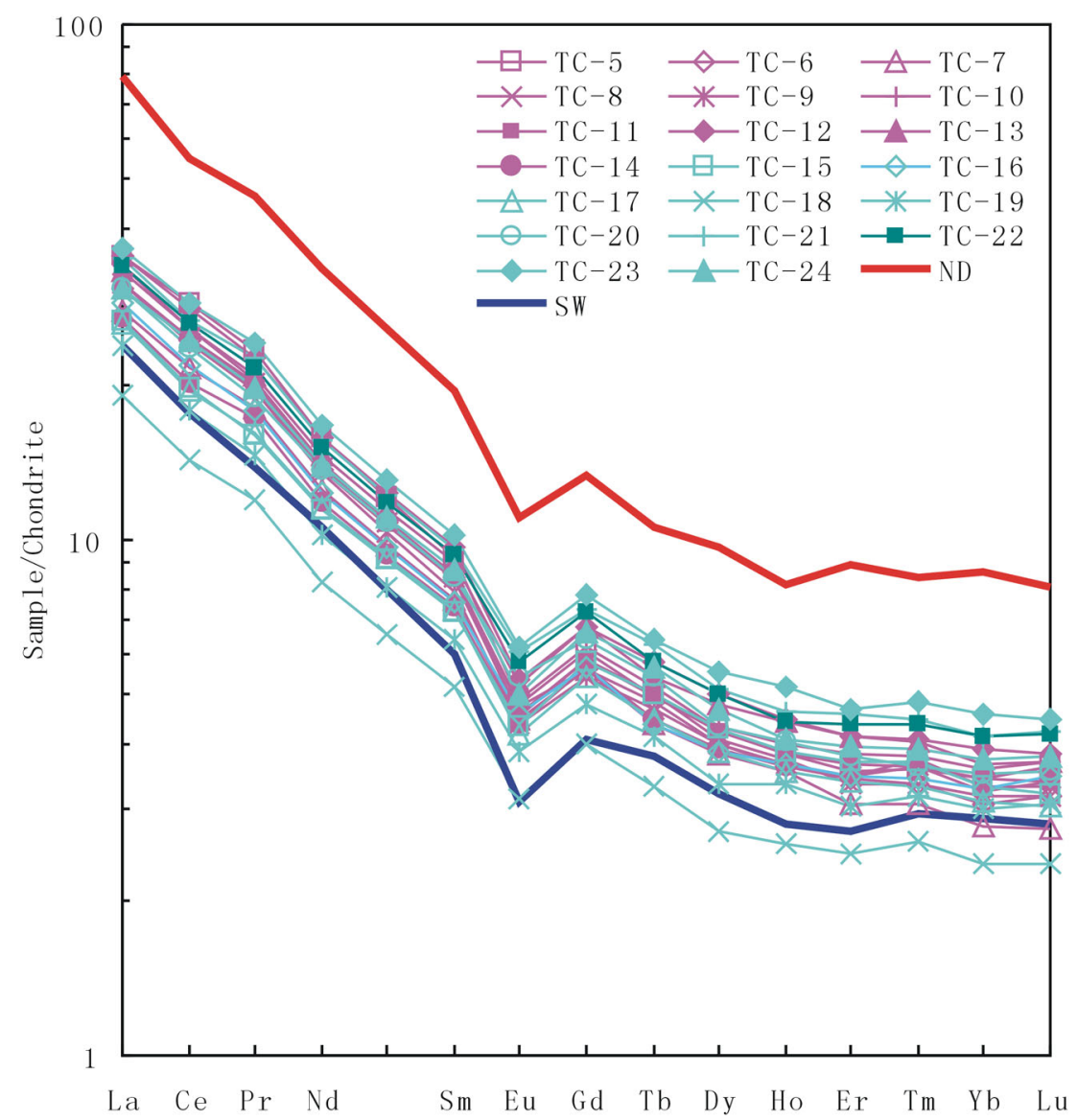

Fig. 6. Distribution patterns of rare earth elements in oil shale samples from the Shengli River area. Note the similarity in REE patterns for oil shale samples, the Nadi Kangri felsic volcanic rocks (ND) and the Suowa Formation limestone (SW).

The Suowa Formation limestone has low concentration of REEs (8.32-78.48 $\mu \mathrm{g} / \mathrm{g}$, average $41.16 \mu \mathrm{g} / \mathrm{g}$ ) and shows clearly fractionated LREEs relative to HREEs (LREE/HREE $=6.62-9.22$ ) with a distinct negative Eu anomaly $(0.53-0.75)$. These patterns are generally similar to those of the oil shale samples from the Shengli River area, indicating that part of the REEs in oil shale samples were derived from the Suowa Formation limestone.

The REE, Th and Sc are generally considered to be the most reliable indicators of sediment provenance because their distribution is less affected by heavy-mineral fractionation than that of elements such as $\mathrm{Zr}$, $\mathrm{Hf}$, and $\mathrm{Sn}$ [13]. The ratios such as $\mathrm{Eu} / \mathrm{Eu}^{*},(\mathrm{La} / \mathrm{Lu})_{\mathrm{N}}, \mathrm{La} / \mathrm{Sc}, \mathrm{Th} / \mathrm{Sc}$, and $\mathrm{Cr} / \mathrm{Th}$ are significantly different in mafic and felsic source rocks and can, therefore, provide information about the provenance of sedimentary rocks [23]. The $\mathrm{Eu} / \mathrm{Eu}^{*}\left(\mathrm{Eu} / \mathrm{Eu}^{*}=\right.$ europium anomaly calculated as 
$\left.\left[\mathrm{Eu} /\left(\mathrm{Eu}^{*}\right)\right]=\left[\left(\mathrm{Eu}_{\mathrm{N}}\right) /\left(\left(\mathrm{Sm}_{\mathrm{N}}+\mathrm{Gd}_{\mathrm{N}}\right) / 2\right)\right]\right),(\mathrm{La} / \mathrm{Lu}) \mathrm{N}, \mathrm{La} / \mathrm{Sc}, \mathrm{Th} / \mathrm{Sc}$, and $\mathrm{Cr} / \mathrm{Th}$ ratios of the oil shale samples are similar to those of the Nadi Kangri felsic volcanic source rocks and the Suowa Formation limestone (Table 4), indicating that elements related to terrigenous origin in the oil shale samples were mainly derived from the Nadi Kangri felsic volcanic rocks and the Suowa Formation limestone in the Qiangtang basin.

Table 4. Range of elemental ratios of the oil shale samples compared to the ratios in the Nadi Kangri Formation felsic volcanic rocks, the Suowa Formation limestone and Upper Continental Crust

\begin{tabular}{|c|c|c|c|c|}
\hline $\begin{array}{l}\text { Elemental } \\
\text { ratio }\end{array}$ & $\begin{array}{l}\text { Range of oil } \\
\text { shale }^{\mathrm{a}}\end{array}$ & $\begin{array}{c}\text { Range of the Nadi } \\
\text { Kangri Formation } \\
\text { felsic volcanic } \\
\text { rocks }\end{array}$ & $\begin{array}{l}\text { Range of the } \\
\text { Suowa } \\
\text { Formation } \\
\text { limestone }\end{array}$ & $\begin{array}{c}\text { Upper } \\
\text { Continental } \\
\text { Crust }^{\mathrm{c}}\end{array}$ \\
\hline $\mathrm{La} / \mathrm{Sc}$ & $\begin{array}{c}1.81-2.46 \\
\text { (average 2.01) }\end{array}$ & $\begin{array}{c}1.62-4.22 \\
\text { (average 2.72) }\end{array}$ & $\begin{array}{c}0.25-2.49 \\
\text { (average 1.25) }\end{array}$ & 1.91 \\
\hline $\mathrm{Th} / \mathrm{Sc}$ & $\begin{array}{c}0.66-0.85 \\
\text { (average } 0.76 \text { ) }\end{array}$ & $\begin{array}{c}0.55-1.14 \\
\text { (average } 0.80 \text { ) }\end{array}$ & $\begin{array}{c}0.09-0.89 \\
\text { (average } 0.40 \text { ) }\end{array}$ & 0.71 \\
\hline $\mathrm{Cr} / \mathrm{Th}$ & $\begin{array}{c}6.91-9.49 \\
\text { (average 8.58) }\end{array}$ & $\begin{array}{c}0.63-9.67 \\
\text { (average 3.76) }\end{array}$ & $\begin{array}{c}7.02-41.37 \\
\text { (average 18.46) }\end{array}$ & 4.46 \\
\hline $\mathrm{Eu} / \mathrm{Eu}^{*}$ & $\begin{array}{c}0.63-0.72 \\
\text { (average } 0.67 \text { ) }\end{array}$ & $\begin{array}{c}0.42-0.98 \\
\text { (average } 0.68 \text { ) }\end{array}$ & $\begin{array}{c}0.53-0.75 \\
\text { (average } 0.63 \text { ) }\end{array}$ & 0.59 \\
\hline$(\mathrm{La} / \mathrm{Yb})_{\mathrm{N}}$ & $\begin{array}{c}7.88-10.37 \\
\text { (average } 8.87 \text { ) }\end{array}$ & $\begin{array}{c}5.64-16.24 \\
\text { (average 10.09) }\end{array}$ & $\begin{array}{c}7.28-10.81 \\
\text { (average } 8.93 \text { ) }\end{array}$ & \\
\hline
\end{tabular}

a This study; ${ }^{\mathrm{b}}$ Fu et al.[22]; ${ }^{\mathrm{c}}$ Condie [24]

\section{Conclusions}

1. On the average, the most abundant trace elements in the oil shale seams are $\mathrm{Sr}(407.2-2365.2 \mu \mathrm{g} / \mathrm{g})$ and $\mathrm{Ba}(85.84-256.3 \mu \mathrm{g} / \mathrm{g})$. All the other elements occur in amounts smaller than $100 \mu \mathrm{g} / \mathrm{g}$. The concentrations of $\mathrm{Sr}, \mathrm{U}, \mathrm{Rb}, \mathrm{Pb}$, and $\mathrm{Th}$ are 1.22 to 3.66 times higher compared to the average concentrations in the crust (Clarke values), while $\mathrm{Co}, \mathrm{Cr}, \mathrm{Sc}, \mathrm{Dy}$, $\mathrm{Er}, \mathrm{Eu}, \mathrm{Ga}, \mathrm{Hf}, \mathrm{Ho}, \mathrm{Lu}, \mathrm{Nb}, \mathrm{Sc}, \mathrm{Ta}, \mathrm{Tb}, \mathrm{V}, \mathrm{Y}, \mathrm{Yb}, \mathrm{Zn}$, and $\mathrm{Zr}$ are decreasing. Clearly, the abundance or concentration of an element may be a function of that association and the origins of the various oil shale fractions.

2. The elements in the Shengli River oil shale may be classified by cluster analysis into three groups. The first group (organic sulphur, total sulphur, $\mathrm{U}, \mathrm{V}, \mathrm{Fe}, \mathrm{Pb}, \mathrm{Na}, \mathrm{Cu}, \mathrm{Ni}$, organic carbon, and $\mathrm{Co}$ ) comprises elements with positive correlation with the organic carbon content. The second group $(\mathrm{Mn}, \mathrm{Sr}, \mathrm{Ca}$, and $\mathrm{Mg}$ ) has weakly positive or slightly negative correlation coefficients with ash yield, while the third group (Dy, Ho, Tb, $\mathrm{Y}, \mathrm{Tm}, \mathrm{Yb}, \mathrm{P}, \mathrm{Eu}, \mathrm{Gd}, \mathrm{Er}, \mathrm{Lu}, \mathrm{Nd}, \mathrm{Pr}, \mathrm{Ga}, \mathrm{Sm}, \mathrm{Ce}, \mathrm{La}, \mathrm{Ge}, \mathrm{Zn}, \mathrm{Ba}, \mathrm{Si}, \mathrm{K}$, 
$\mathrm{Cr}, \mathrm{Nb}, \mathrm{Zr}, \mathrm{Rb}, \mathrm{Th}, \mathrm{Hf}, \mathrm{Ta}, \mathrm{Sc}$, and $\mathrm{Ti}$ ) shows high positive correlation coefficients with ash yield.

3. In the Shengli River oil shale, elements related to terrigenous origin in the oil shale seams have originated from two sources: one is the Nadi Kangri Formation felsic volcanic rock source and the other is the Suowa Formation limestone.

\section{Acknowledgements}

This work was supported by the Sichuan Youth Science \& Technology Foundation (No. 09ZQ026-006), the National Natural Science Foundation of China (No. 40702020, 40972087) and the National Oil and Gas Special Project (No.XQ-2009-01).

\section{REFERENCES}

1. Dyni, J. R. Oil shale developments in the United States// Oil Shale. 2006. Vol. 23, No. 2. P. 97-98.

2. Kök, M. V. Oil shale resources in Turkey// Oil Shale. 2006. Vol. 23, No. 3. P. 209-210.

3. Liu, Z. J., Yang, H. L., Dong, Q.S., Zhu, J. W., Guo, W., Ye, S. Q., Liu, R., Meng, Q. T., Zhang, H. L., Gan, S. C. Oil Shale in China. - Beijing: Petroleum Industry Press, 2009. P. 1157-1167 [in Chinese with English abstract].

4. Wang, C. S., Zhang, S. M. The discovery of oil shale in the Shuanghu area, Northern Tibet, China // Geology in China. 1987. No. 8. P. 29-31 [in Chinese].

5. Fu, X. G., Wang, J., Qu, W. J., Duan, T. Z., Du, A. D., Wang, Z. J., Liu, H. ReOs (ICP-MS) dating of marine oil shale in the Qiangtang basin, Northern Tibet, China // Oil Shale. 2008. Vol. 25, No. 1. P. 47-55.

6. Fu, X. G., Wang, J., Zeng, Y. H., Li, Z. X., Wang, Z. J. Geochemical and palynological investigation of the Shengli River marine oil shale (China): implications for paleoenvironment and paleoclimate// Int. J. Coal Geol. 2009. Vol. 78, No. 3. P. 217-224.

7. Fu, X. G., Wang, J., Tan, F. W., Zeng, Y. H. Sedimentological investigations of the Shengli River-Changshe Mountain oil shale (China): relationships with oil shale formation // Oil Shale. 2009. Vol. 26, No. 3. P. 373-381.

8. Fu, X. G., Wang, J., Zeng, Y. H., Tan, F. W., Feng, X. L. REE geochemistry of marine oil shale from the Changshe Mountain area, northern Tibet, China // Int. J. Coal Geol. 2010. Vol. 81, No. 3. P. 191-199.

9. Wang, J., Ding, J., Wang, C. S., Tan, F. W., Chen, M., Hu, P., Li, Y. L., Gao, R., Fang, H., Zhu, L. D., Li, Q. S., Zhang, M. H., Du, B.W., Fu, X. G., Li, Z.X., Wan, $F$. Survey and evaluation on Tibet oil and gas resources. - Beijing: Geological Publishing House, 2009. 6 pp. [in Chinese]. 
10. Kimura, $T$. Relationships between inorganic elements and minerals in coals from the Ashibetsu district, Ishikari coal field, Japan // Fuel Process. Technol. 1998. Vol. 56, No. 1-2. P. 1-19.

11. Liu, Y., Liu, H. C., Li, X. H. Simultaneous and precise determination of 40 trace elements in rock samples using ICP-MS // Geochimica. 1996. Vol. 25, No. 6. P. 552-558 [in Chinese with English abstract].

12. Patterson, J. H., Ramsden, A. R., Dale, L. S., Fardy, J. J. 1986. Geochemistry and mineralogical residences of trace elements in oil shales from Julia Creek, Queensland, Australia // Chem. Geol. 1986. Vol. 55, No. 1-2. P. 1-16.

13. Taylor, S. R., McLennan, S. M. The geochemical evolution of the continental crust // Rev. Geophys. 1995. Vol. 33, No. 2. P. 241-265.

14. Spears, D. A., Zheng, $Y$. Geochemistry and origin of elements in some UK coals // Int. J. Coal Geol. 1999. Vol. 38, No. 3-4. P. 161-179.

15. Eskenazy, G. M. Trace elements geochemistry of the Dobrudza coal basin, Bulgaria // Int. J. Coal Geol. 2009. Vol. 78, No. 3. P. 192-200.

16. Dai,S.F., Li,D., Chou, C.L., Zhao,L., Zhang, Y., Ren,D.Y., Ma,Y.W., Sun, $Y$. $Y$. Mineralogy and geochemistry of boehmite-rich coals: new insights from the Haerwusu Surface Mine, Jungar Coalfield, Inner Mongolia, China// Int. J. Coal Geol. 2008. Vol. 74, No. 3-4. P. 185-202.

17. Mukhopadhyay, P. K., Goodarzi, F., Crandlemire, A. L., Gillis, K. S., MacNeil, D. J., Smith, W. D. Comparison of coal composition and elemental distribution in selected seams of the Sydney and Stellarton Basins, Nova Scotia, Eastern Canada // Int. J. Coal Geol. 1998. Vol. 37, No. 1-2. P. 113-141.

18. Gürdal, G. Geochemistry of trace elements in Çan coal (Miocene), Çanakkale, Turkey // Int. J. Coal Geol. 2008. Vol. 74, No. 1. P. 28-40.

19. Patterson, J. H., Ramsden, A. R., Dale, L. S., Fardy, J. J. Geochemistry and mineralogical residences of trace elements in oil shales from Julia Creek, Queensland, Australia // Chem. Geol. 1986. Vol. 55, No. 1-2. P. 1-16.

20. Vassilev, S. V., Vassileva, C. G. Comparative chemical and mineral characterization of some Bulgarian coals // Fuel Process. Technol. 1998. Vol. 55, No. 1. P. 55-69.

21. Wang, J., Fu, X. G., Chen, W.X., Wang, Z.J., Tan, F. W., $\quad$ Chen, M., Zhuo, J.W. Chronology and geochemistry of the volcanic rocks in Woruo Mountain region, Northern Qiangtang depression: implications to the Late Triassic volcanic-sedimentary events // Sci. China Ser. D. 2008. Vol. 51, No. 2. P. 194-205.

22. Fu, X. G., Wang, J., Tan, F. W., Chen, M., Chen, W. B. The Late Triassic riftrelated volcanic rocks from eastern Qiangtang, northern Tibet (China): Age and tectonic implications // Gondwana Res. 2010. Vol. 17, No. 1. P. 135-144.

23. Amstrong-Altrin, J. S., Lee, Y. I., Verma, S. P., Ramasamy, S. Geochemistry of sandstones from the Upper Miocene Kudankulam Formation, Southern India: implications for provenance, weathering and tectonic setting // J. Sediment. Res. 2004. Vol. 74, No. 2. P. 285-297.

24. Condie, $K$. C. Chemical composition and evolution of the upper continental crust: contrasting results from surface samples and shales // Chem. Geol. 1993. Vol 104, No. 1-4. P. 1-37.

Presented by Jialin Qian

Received January 20, 2011 